\title{
An Independently Tunable Tri-band Antenna Design for Concurrent Multi-band Single Chain Radio Receivers
}

\author{
Q. Bai, R. Singh, Member, IEEE, K. L. Ford, Senior Member, IEEE, T. O'Farrell, Member, IEEE, \\ and R. J. Langley, Senior Member, IEEE
}

\begin{abstract}
In this paper, a novel tunable tri-band antenna is presented for concurrent, multi-band, single chain radio receivers. The antenna is manufactured on a $50 \times 100 \mathrm{~mm}$ FR4 printed circuit board (PCB), and is able to provide three concurrent, independently tunable operating bands covering a frequency range from $600 \mathrm{MHz}$ to $2.7 \mathrm{GHz}$. The antenna performance is investigated for both numerical and experimental methods when using, first, varactor diodes and, second, digitally tunable capacitors (DTCs) to tune frequencies, which shows the antenna gain can be improved by up to $2.6 \mathrm{dBi}$ by using DTCs. A hardware-in-the-loop test-bed provides a system level evaluation of the proposed antenna in a direct RF digitized, concurrent, tri-band radio receiver. By measuring the receiver's error vector magnitude, we demonstrate sufficient isolation between concurrent bands achieving $30 \mathrm{MHz}$ of aggregated bandwidth as well as strong resilience to adjacent blockers next to each band. The data reported in this article are available from the ORDA digital repository (https://doi.org/10.15131/shef.data.5346295).
\end{abstract}

Index Terms-5G, Reconfigurable antennas, reconfigurable architectures, system analysis and design, tunable circuits and devices, radio receivers, multiple band antennas, digital radio, communication systems, cellular radio

\section{INTRODUCTION}

$\mathbf{T}$ HE growing user traffic on radio access networks (RANs) requires the fifth generation $(5 \mathrm{G})$ of cellular mobile radio access technologies (RATs) to have higher area capacities, lower latencies and greater energy efficiency [1]. Moreover, the new 5G RANs are expected to be highly heterogeneous and ultra dense while operating both legacy and new RATs across diverse regions of the RF spectrum [2] [3]. In particular, 5G RATs will support user equipment (UE) with multiple concurrent connections to access nodes and diverse modes of carrier-aggregation (CA) and cognitive radio (CR) techniques. Instead of using multi-chain transceiver systems, one novel solution is to develop a single chain, concurrent, multi-band, frequency-agile radio (CM-FARAD) system, which is also the focus of the UK EPSRC FARAD (Frequency Agile Radio) project [4].

To support such a CM-FARAD system, the antenna needs to provide multiple frequency bands simultaneously, with each band being independently tunable over the desired frequency range. Various methods and techniques have been reported to make the antenna resonant frequency tunable. For example, by using switches or PIN diodes to: change the antenna geometry and/or alter the antenna surface current distribution [5][6]; tune the antenna radiation element impedance by incorporating tunable capacitors [7][8]; vary the antenna substrate properties by using ferrite materials [9] or liquid crystals [10]; or employ tunable matching circuits for non-resonant antenna structures [11]. By using one or combining several tuning methods, a single antenna unit can provide one or more narrowband channels that are tunable over a wide frequency range. A number of tunable dual-band antennas have been reported for various antenna types. In [7], a planar inverted F-antenna (PIFA) was presented to provide two tunable bands over the frequency range from $88 \mathrm{MHz}$ to $2175 \mathrm{MHz}$. In [8], a dualband folded slot antenna was proposed to cover a wide tuning range from 1 to $3 \mathrm{GHz}$. A tunable L-shape patch antenna was investigated in [12] for GSM/DSC-1800 systems.

However, the previous research did not address comprehensively the independent tuning of dual-band antenna designs. One of the limiting factors is the mutual coupling between radiating elements, which can manifest itself as an unwanted shift in the antenna resonant frequency due to the tuning of another band. This will have a detrimental impact on the system performance, as often the antennas are narrowband over the frequencies of interest. The isolation between two tunable frequency bands can be improved by using dualfeeding structures [13], which is not suitable for a single chain transceiver. In [14], independent tuning was obtained by using a coplanar waveguide (CPW) feeding network, which will significantly increase the complexity of the antenna geometry especially when the number of antenna operating bands increases.

We propose a compact, tunable, tri-band antenna design for a frequency agile, concurrent multi-band (CM) receiver. The antenna supports three independent, continuously tunable operating bands covering a frequency range from $600 \mathrm{MHz}$ to $2.7 \mathrm{GHz}$. Three tunable capacitors are used in the design as the active tuning components. In a first design, varactor diodes are used as tuning components whereas in a second design digitally tunable capacitors are used. The two designs are compared based on their antenna radiation performances. The antennas were manufactured on a $50 \times 100 \mathrm{~mm}$ FR4 PCB with a planar structure, which provides a low profile and low cost antenna solution suitable for modern mobile handsets.

We evaluate the system performance of a direct RF digitizing, concurrent, tri-band, single chain radio receiver testbed. An investigation into inter-band interference (IBI) between the concurrent transmissions and adjacent/co-located channel interference is carried out for a heterogeneous network (HetNet) 
scenario based on tuning a CM-FARAD UE to 3 distinct transmissions representing a macro-cell and two small-cell BTSs.

\section{Concurrent Multi-Band Radio Architectures}

The radio architecture being developed is shown in Fig.1. The CM-FARAD architecture is a single-chain radio system where both the analog front-end and digital processing are capable of frequency-agile CM operation. That is, analog subsystems, such as antenna, filters, PA and LNA must concurrently receive and transmit over multiple frequency-agile bands. Further, digital processing such as analog-to-digital conversion (ADC), digital-to-analog conversion (DAC), digital down-conversion (DDC) and digital up-conversion (DUC) are also reconfigurable with $\mathrm{CM}$ capability. In order to design the CM-FARAD system, enabling CM operation at the radio subsystem level as well as direct RF digitization is critical.

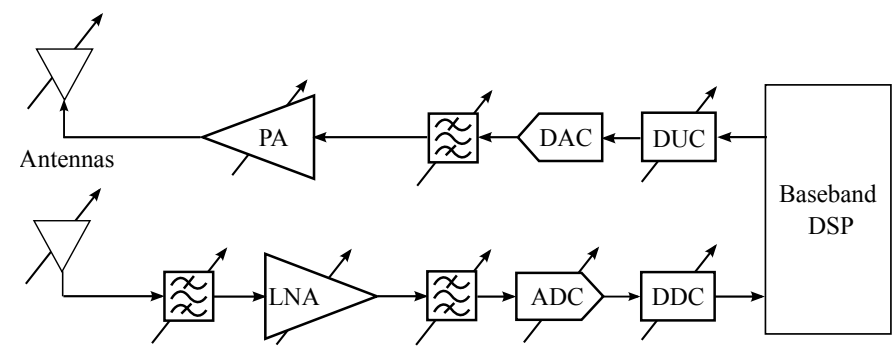

Fig. 1. The architecture of a direct RF conversion concurrent multi-band frequency-agile radio (CM-FARAD).

CM capable radio sub-systems have been under investigation for some time now. In the analog domain, along with the multi-band tunable antennas [7][8], various designs for dualband power amplifiers (PAs) [15] and low noise amplifiers (LNAs) [16] have been presented. Enabling tunability along with multi-band operation has proved challenging, especially at the PA [15]. However, for the LNA, adjacent blocker mitigation is another key concern [17]. Multi-band reconfigurable ADCs have also been designed to support intra-band carrier aggregation [18]. However, a power efficient and small size solution for $\mathrm{CM}$ digitization as desired in CM-FARAD, is currently not available and so needs to be researched.

Despite the recent developments in radio sub-systems to enable $\mathrm{CM}$ operation, the solution for CM frequency-agile radios is still an open question. The analogue mixing homodyne (direct-conversion) radio architecture, which is widely used in software-defined radio (SDR) systems [19][20] and the multistandard, multi-chain mobile transceiver chipset [21][22], use one transceiver chain, requiring one antenna (which might be frequency-agile) for each communication standard.

Given the requirements of 5G RANs and the expected increase in the number of RATs working concurrently, the multi-standard transceiver chipset and the SDR systems, which are used in the UEs and at the BTSs, respectively, must accommodate more than one standard or communication link per transceiver chain. Therefore, the development of $\mathrm{CM}$ tunable antennas becomes essential to providing multiple, independently tunable, operating bands, whilst having a compact profile to fit into a modern UE.

\section{Antenna Design}

\section{A. Antenna Geometry and Design Features}

The configuration of the proposed antenna is presented in Fig. 2. The antenna consists of three slots on a PCB ground plane which are resonant at three concurrent frequency bands. As shown in Fig. 2(a), two slots are placed parallel to the Xaxis with a $4 \mathrm{~mm}$ separation and located $g_{1}=6 \mathrm{~mm}$ away from the PCB top edge. A Y-axis orientated slot of length $S l_{2}=15 \mathrm{~mm}$, is placed in between the $\mathrm{X}$-axis orientated slots spaced $g_{2}=2 \mathrm{~mm}$ away from the PCB top edge. All three slots are driven by a single microstrip line through electromagnetic coupling, which is printed on the back of the PCB, as shown in Fig. 2(b). Each slot includes a tunable capacitor to enable frequency agility. A close up view of the proposed slots and the location of tunable capacitors is shown in Fig. 2(c). The antenna measures $50 \times 100 \mathrm{~mm}(W \times L)$, which is double sided on an $1.6 \mathrm{~mm}$ thick FR4 substrate $\left(\varepsilon_{r}=4.3\right.$, loss tangent $\left.=0.025\right)$. More details of the antenna geometry are given in Table I.

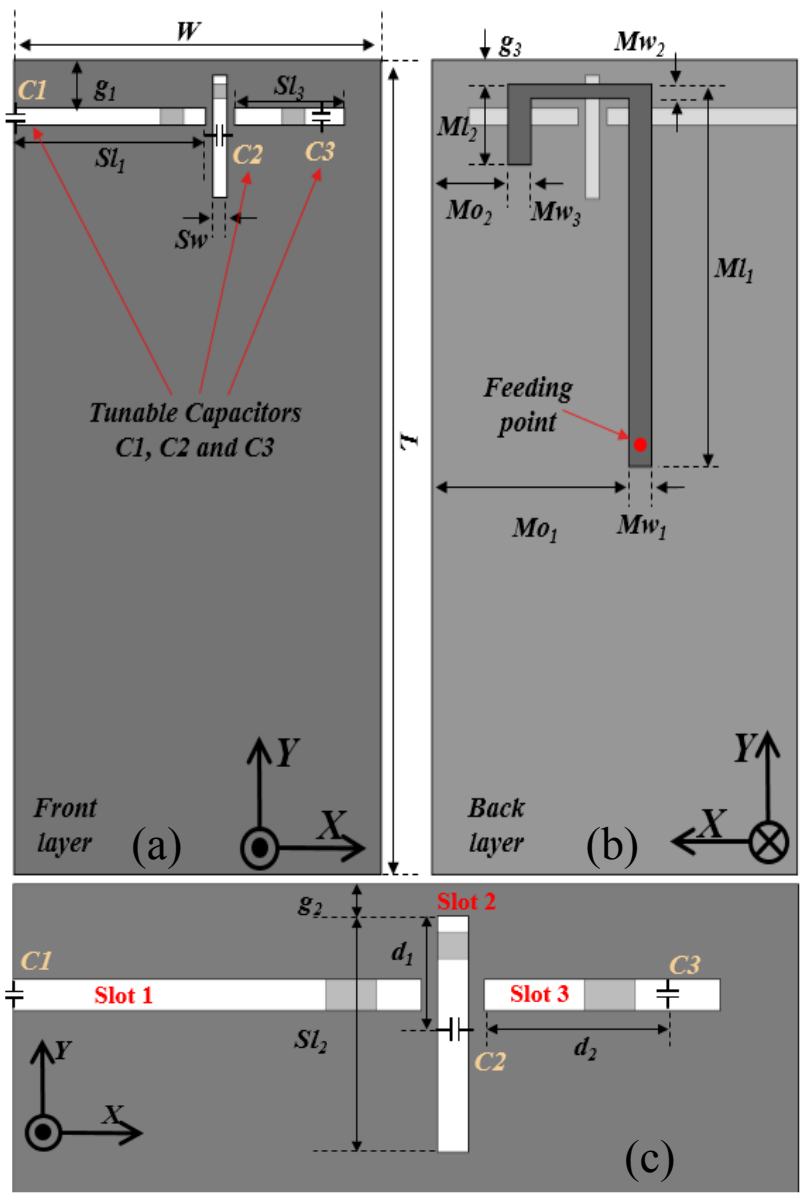

Fig. 2. Tunable, multiband antenna geometry: (a) front layer, (b) back layer, (c) tunable capacitor locations

Slot antennas have been extensively studied in [23], where the slot at its first mode can be considered as a $\lambda / 2$ transmission line short circuited at two ends. The slot length can be further reduced to $\lambda / 4$ if one end of the slot is open circuited, which is known as an open-end slot. According to 
TABLE I

ANTENNA CONFIGURATION PARAMETERS

\begin{tabular}{|c|c|c|c|c|c|}
\hline Parameter & $W \times L$ & $S l_{1}$ & $S_{2}$ & $S l_{3}$ & $S w$ \\
\hline Value (mm) & $50 \times 100$ & 26 & 15 & 15 & 2 \\
\hline \hline Parameter & $g_{1}$ & $g_{2}$ & $g_{3}$ & $M w_{1}$ & $M w_{2}$ \\
\hline Value (mm) & 6 & 2 & 3 & 3.2 & 1.7 \\
\hline \hline Parameter & $M w_{3}$ & $M o_{1}$ & $M o_{2}$ & $M l_{1}$ & $M l_{2}$ \\
\hline Value (mm) & 3.2 & 26.9 & 10.4 & 47 & 10 \\
\hline \hline Parameter & $d_{1}$ & $d_{2}$ & & & \\
\hline Value (mm) & 7.5 & 12 & & & \\
\hline
\end{tabular}

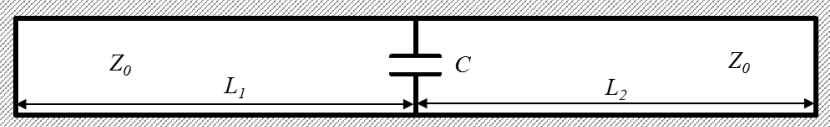

Fig. 3. Transmission line model of slot antenna with lumped capacitor $(C)$

Babinet's principle, the radiation pattern of the slot antenna will be identical in shape to its complementary dipole but with interchanged $\mathrm{E}$ and $\mathrm{H}$ fields [24]. As shown in Fig. 3, the slot line capacitance can be increased by adding a capacitor $(C)$. The resonant frequency of the slot is then determined by, not only the slot impedance, but also the location and value of the capacitor. From [8], if not considering the effect of the feed, the resonant frequency of the slot shown in Fig. 3 can be calculated by (1).

$$
\begin{aligned}
& \tan \left(\beta(\omega) L_{1}\right)+\tan \left(\beta(\omega) L_{2}\right) \\
& \quad-\omega C Z_{0} \tan \left(\beta(\omega) L_{1}\right) \tan \left(\beta(\omega) L_{2}\right)=0
\end{aligned}
$$

where $C$ is the capacitance of the loaded capacitor, $L_{1}$ and $L_{2}$ are the inductances of the partitioned slot, $\omega$ is the slot resonant frequency, $\beta(\omega)$ is the slot line propagation constant, and $Z_{0}$ is the slot line impedance. From (1), we can see that the resonant frequency of the slot antenna can be shifted by tuning the capacitance of the loaded capacitor if the slot line impedance and the capacitor location are fixed.

Three tunable slots are used in this antenna design as the basic radiation element to achieve three concurrent tunable frequency bands covering the range from 0.6 to $2.7 \mathrm{GHz}$. Due to the limited tuning range of a single slot, the desired system frequency range is divided into three sub-ranges: 0.6 to 1.1 $\mathrm{GHz}$ (low range), 1 to $2.5 \mathrm{GHz}$ (middle range) and 1.9 to 2.7 $\mathrm{GHz}$ (high range), which are covered by slots 1, 2 and 3 in Fig. 2(c), respectively. One of the key objectives of this tunable tri-band antenna design is to achieve independent tunability of each band, which means tuning any one slot of the antenna should not affect the other two slots. The requirement for a low antenna profile in modern mobile handsets, where the internal space is extremely limited, makes this particular task difficult. Therefore, the location and polarisation of each slot needs to be carefully chosen in order to minimise the mutual coupling between radiating elements.

The electromagnetic software package, CST Microwave Studio, was used to model the antenna. Fig. 4 shows the

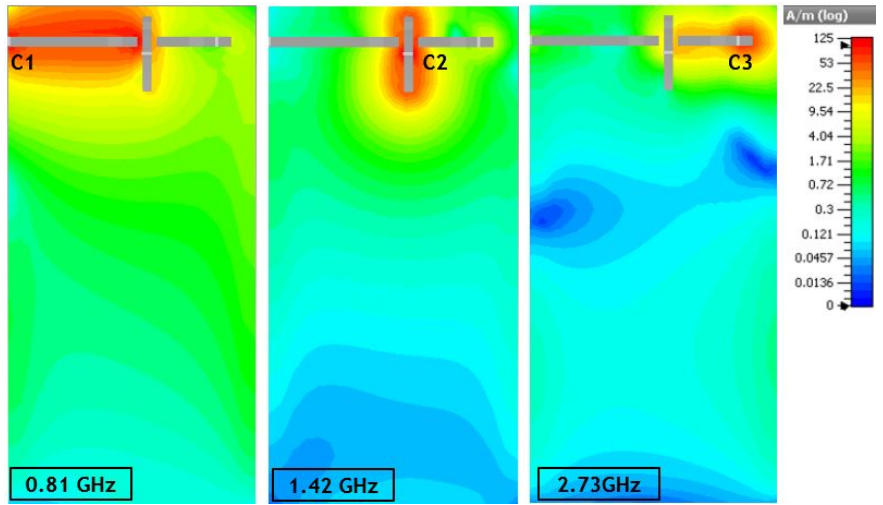

Fig. 4. Antenna surface current distribution at $0.81 \mathrm{GHz}, 1.42 \mathrm{GHz}$ and $2.73 \mathrm{GHz}$ (all 3 bands use the same colour bar scale)

antenna surface current distributions for the three concurrent frequency bands when slots 1,2 and 3 resonate at 0.81 , 1.42 and $2.73 \mathrm{GHz}$, respectively. The figure gives a direct visualisation of the operating characteristics of each slot. At $0.81 \mathrm{GHz}$, Fig. 4 illustrates that strong surface currents are only found around slot 1 , which indicates that slot 1 is the main resonant element in the low band. Slot 3 is in line with slot 1 with only a $4 \mathrm{~mm}$ gap between them. Normally, such a parallel-slot structure leads to strong mutual coupling, which can be utilized to produce additional resonant frequency bands [25], though this affects the independent tunability of each slot. Therefore, a short vertical slot (slot 2) is placed between slots 1 and 3 to reduce the unwanted coupling. The coupling caused by inserting slot 2 is also minimised due to the electromagnetic field of this vertical slot being cross-polarised to the fields of slots 1 and 3. The lumped capacitor (C3) on slot 3 is placed on the right hand side of the slot to further decrease the surface current strength on its left hand side, which is closer to slot 1 , thereby further reducing any mutual coupling.

\section{B. Antenna Fabrication and Experimental Measurements}

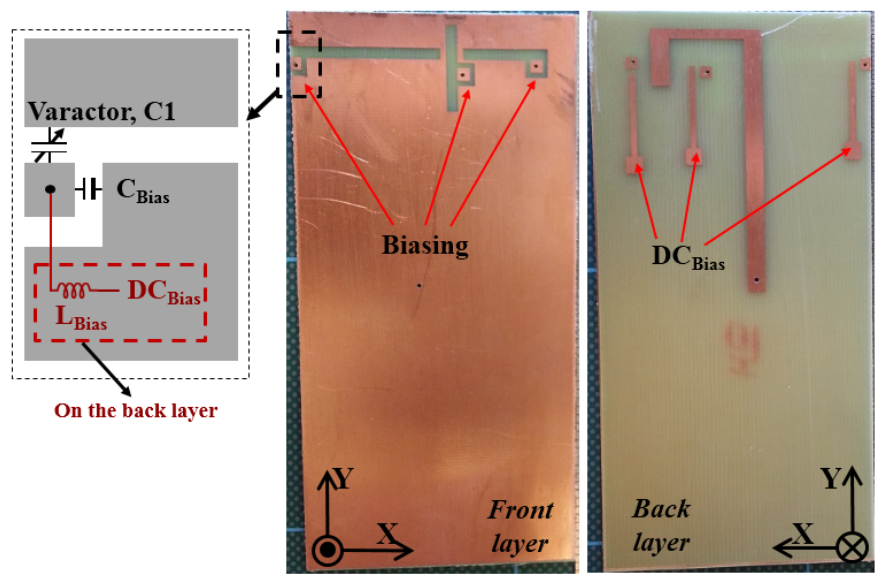

Fig. 5. Manufactured antenna with varactors and biasing network

The antenna was manufactured on a $1.6 \mathrm{~mm}$ FR4 substrate, and both varactor diodes and DTCs were used as the tunable lumped components on the slots, which were biased separately 

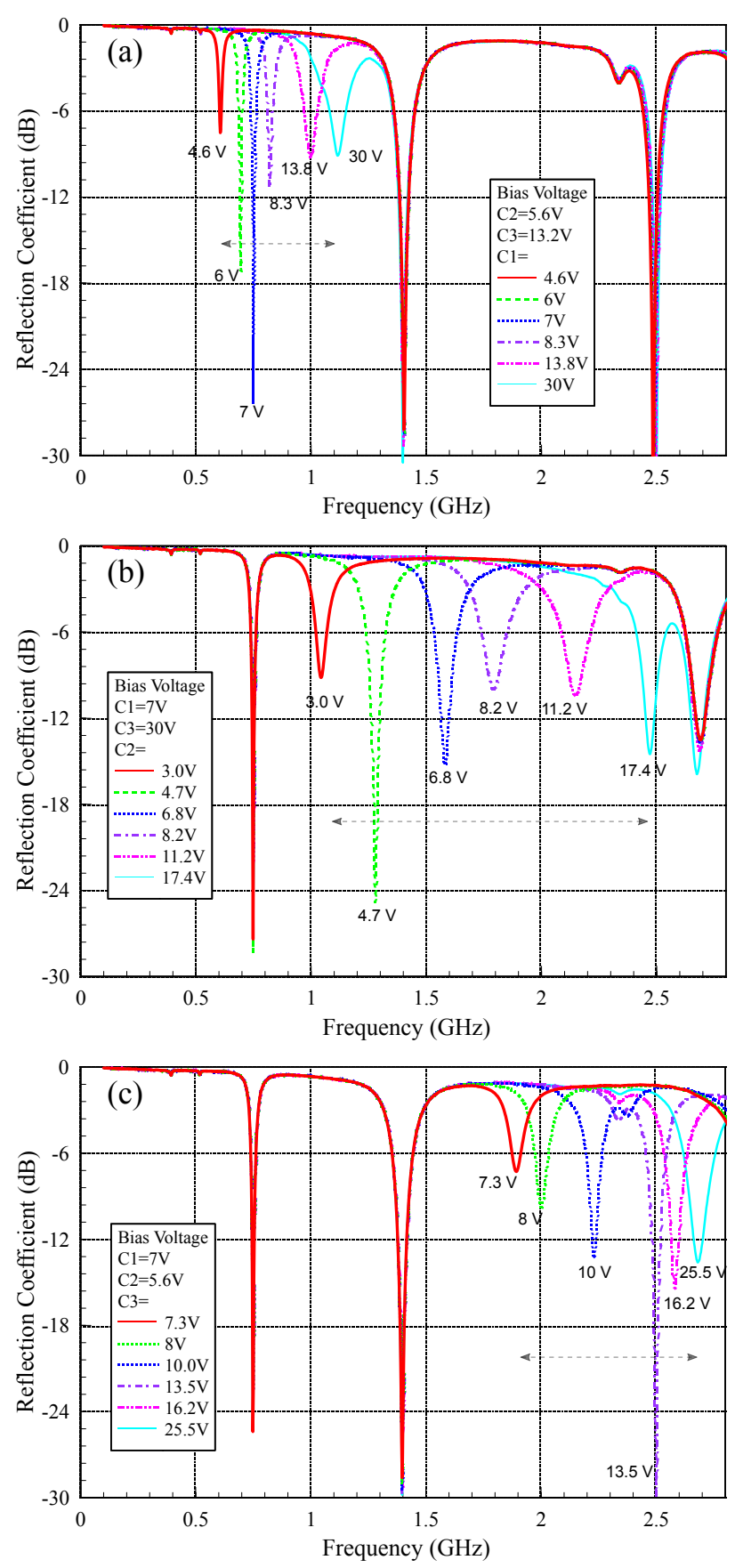

Fig. 6. Measured antenna reflection coefficients: (a) tuning $\mathrm{C} 1$, (b) tuning $\mathrm{C} 2$, (c) tuning $\mathrm{C} 3$

by three power supplies. The varactor used in the antenna design is model E6327 from Infineon, which has a capacitance tuning range from $0.6 \mathrm{pF}$ to $10 \mathrm{pF}$ with a bias voltage up to 30 V. As shown in Fig. 5, biasing networks were designed with capacitors and inductors acting as blockers to reduce the interference between the antenna feeding source and the DC biasing currents.

The measured antenna reflection coefficients under various biasing voltages are plotted in Fig. 6. The resonant frequency of the low band can be tuned by changing the bias voltage of the varactor diode $(C 1)$ mounted on slot 1 . The $-6 \mathrm{~dB}$ reflection coefficient tuning range covers from $600 \mathrm{MHz}$ to
TABLE II

SIMULATED AND MEASURED ANTENNA TUNING CHARACTERISTICS FOR THE THREE BANDS

\begin{tabular}{|c|c|c|c|c|c|}
\hline \multirow{2}{*}{} & \multirow{2}{*}{$\begin{array}{c}\text { Bias Voltage } \\
(\mathrm{V})\end{array}$} & \multicolumn{2}{|c|}{$\begin{array}{c}\text { Resonant Frequency } \\
(\mathrm{GHz})\end{array}$} & \multicolumn{2}{c|}{$\begin{array}{r}-6 \mathrm{~dB} \text { Bandwidth } \\
(\mathrm{MHz})\end{array}$} \\
\cline { 2 - 6 } & & Simulated & Measured & Simulated & Measured \\
\hline \multirow{3}{*}{ C1 } & 4.6 & 0.61 & 0.60 & 8 & 9 \\
\cline { 2 - 6 } & 8.3 & 0.84 & 0.82 & 24 & 26 \\
\cline { 2 - 6 } & 30.0 & 1.20 & 1.12 & 72 & 75 \\
\hline \hline \multirow{3}{*}{ C2 } & 3.0 & 1.12 & 1.04 & 49 & 49 \\
\cline { 2 - 6 } & 8.2 & 1.81 & 1.80 & 115 & 119 \\
\cline { 2 - 6 } & 17.4 & 2.51 & 2.47 & 126 & 131 \\
\hline \hline \multirow{3}{*}{ C3 } & 7.3 & 1.90 & 1.89 & 35 & 40 \\
\cline { 2 - 6 } & 13.5 & 2.55 & 2.50 & 90 & 94 \\
\cline { 2 - 6 } & 25.5 & 2.72 & 2.70 & 132 & 140 \\
\hline
\end{tabular}
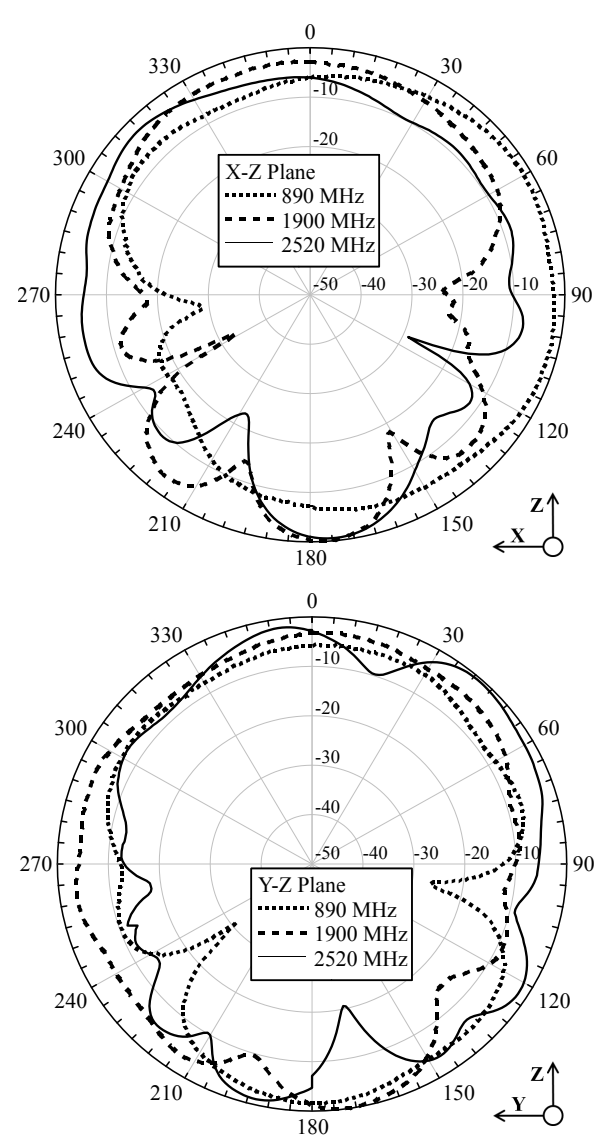

Fig. 7. Measured normalised varactor antenna radiation patterns: top $X-Z$ plane; bottom Y-Z plane

$1.1 \mathrm{GHz}$ (Fig. 6(a)) when varying the bias voltage from $4.6 \mathrm{~V}$ to $30 \mathrm{~V}$. It is important that during the tuning of the low band, the middle and high band resonant frequencies at $1.4 \mathrm{GHz}$ and $2.5 \mathrm{GHz}$, respectively, are unaffected. In order to find out the maximum tuning range of the middle band, the low and high bands were shifted to $750 \mathrm{MHz}$ and $2.7 \mathrm{GHz}$, respectively. As shown in Fig. 6(b), the middle band antenna can be readily tuned from $1.05 \mathrm{GHz}$ to $2.5 \mathrm{GHz}$ by varying the bias voltage from $3 \mathrm{~V}$ to $17.4 \mathrm{~V}$. We observed that the high band resonance at $2.7 \mathrm{GHz}$ starts to be affected when the middle band is 
tuned to $2.5 \mathrm{GHz}$. This indicates that the minimum separation between two operating bands for this antenna is about 200 MHz. That is, strong mutual coupling was obtained when two bands were tuned closer than $200 \mathrm{MHz}$. The high band tuning range is plotted in Fig. 6(c), which shows slot 3 resonating between $1.9 \mathrm{GHz}$ and $2.7 \mathrm{GHz}$ corresponding to bias voltages of $7.3 \mathrm{~V}$ and $25.5 \mathrm{~V}$, respectively. The measured results show good agreement with the simulations. More details of the bias voltages, corresponding resonant frequencies and -6 dB bandwidths are listed in Table II.

Fig. 7 plots measured normalized radiation patterns for the varactor antenna for the three concurrent operating bands at $890 \mathrm{MHz}, 1.9 \mathrm{GHz}$ and $2.52 \mathrm{GHz}$. The plots are normalized with respect to the maximum value observed over the three bands and represent typical radiation patterns for each radiating slot within its frequency tuning range. Mutual interactions of adjacent slots and their close proximity to the PCB edge are the likely cause of the deep notches in the radiation patterns. The simulated and measured total efficiencies of the varactor antenna are about $20 \%$ at $800 \mathrm{MHz}$. The measured antenna gains at these frequencies are given in Table III. These three frequencies are also the operating bands investigated in the system case study presented in Section IV.

Recently, DTCs have been used in antenna designs as they provide enhanced power handling capabilities and relatively low internal losses. In order to improve the antenna radiation performance, DTCs were employed in this research by replacing the high loss varactor diodes. The DTC component used was the STPTIC-39G2 from STMicroelectronics, which has a capacitance tuning range from $0.72 \mathrm{pF}$ to $5.04 \mathrm{pF}$ with a bias voltage up to 24 V. Fig. 8 illustrates the PCB layout of the antenna when loaded with DTCs. When comparing the structure of the slot antennas with DTCs to that using varactor diodes, a fine wire is used to connect the DTCs and the slots because of the very small form factor of the DTC package $-0.61 \times 0.66 \times 0.3 \mathrm{~mm}$. Consequently, the frequency tuning range of the DTC slot antenna is shifted downwards by approximately $100 \mathrm{MHz}$ owing to the small additional inductance introduced by the fine wire.

It was observed that the radiation patterns were very similar for the varactor and DTC antennas, hence only measured gains are reported for brevity. The low internal resistance of the DTC impacts the gain but not the directivity due to the change in antenna efficiency. The gains for the DTC and varactor antennas are compared in Table III where we observe that the measured antenna gain can be improved by $0.51-2.6 \mathrm{~dB}$ with DTCs. The measured antenna gain can also be improved by $4.5 \mathrm{~dB}$ at $600 \mathrm{MHz}$ when using a fixed value capacitor to replace the lossy varactor diode. Also, by employing low loss substrate materials, such as the RT/Duroid series rather than FR4, the simulated antenna gain increases by $1.5 \mathrm{~dB}$, which indicates a total possible $6 \mathrm{~dB}$ gain improvement over the varactor components and FR4 materials at $600 \mathrm{MHz}$. In [7] a tunable dual-band handset PIFA antenna design had a measured gain of $0 \mathrm{dBi}$ at $700 \mathrm{MHz}$ and $1.6 \mathrm{dBi}$ at 2200 $\mathrm{MHz}$.

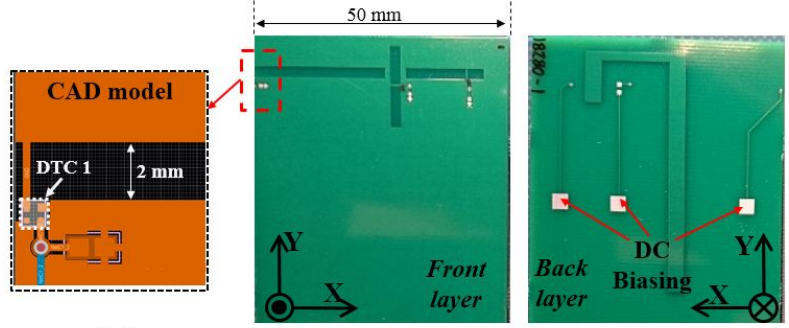

(a)

(b)

Fig. 8. Manufactured antenna with DTCs. (a) the CAD model of DTC which has the dimension of $0.61 \times 0.66 \times 0.3 \mathrm{~mm}$. (b) photos of manufactured antenna

TABLE III

MEASUREd ANTENNA GAIN With VARACTORS AND DTCS

\begin{tabular}{|l|c|r|r|r|r|r|r|}
\hline \multicolumn{2}{|c|}{} & \multicolumn{2}{|c|}{ Slot 1 } & \multicolumn{2}{c|}{ Slot 2 } & \multicolumn{2}{c|}{ Slot 3 } \\
\hline \multicolumn{2}{|c|}{ Frequency $(\mathrm{GHz})$} & 0.70 & 0.89 & 1.40 & 1.90 & 2.00 & 2.52 \\
\hline \multirow{2}{*}{$\begin{array}{c}\text { Gain } \\
\text { (dBi) }\end{array}$} & Varactor & -5.81 & -3.15 & -5.20 & -1.74 & -6.21 & -2.29 \\
\cline { 2 - 8 } & DTC & -3.74 & -2.06 & -4.00 & -0.55 & -5.70 & 0.31 \\
\hline
\end{tabular}

\section{System Level Performance in a HetNet SCENARIO}

In this section, the performance of a tunable, concurrent tri-band, direct RF digitizing, single chain radio receiver (representing an UE), which utilizes the proposed tri-band antenna, is evaluated for 3 concurrent, inter-band transmissions emulating a $5 \mathrm{G}$ HetNet scenario.

We consider a $5 \mathrm{G}$ HetNet scenario which can concurrently connect an UE to a macro-cell BTS and to two small-cell BTSs. The macro-cell BTS operates at $890 \mathrm{MHz}$, whereas the two small-cell BTSs operate at $1900 \mathrm{MHz}$ and $2520 \mathrm{MHz}$, representing an LTE small-cell and a Wi-Fi small cell, respectively. In this test, the CM-FARAD system aims to maintain similar transmission quality across corresponding radio links when operating in concurrent or independent transmission modes. We evaluate the transmission quality of the tri-band receiver using a hardware-in-the-loop test-bed.

\section{A. The Test-bed}

Raw ADC Data (via Ethernet)

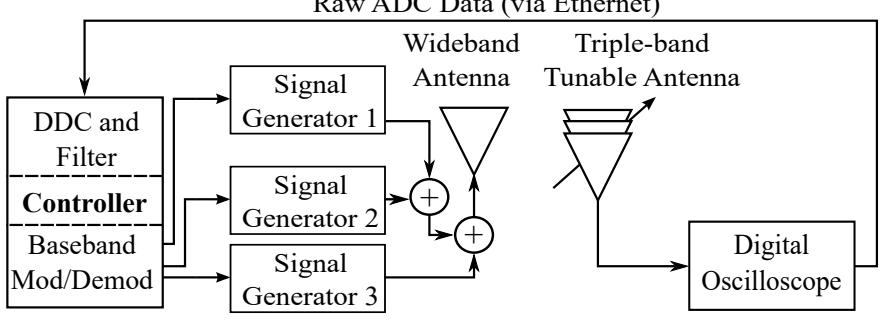

Fig. 9. Schematic of the direct RF digitising tri-band test-bed.

Fig.9 shows a schematic of the test-bed. The controller (PXIe-8135) is a PC running LabVIEW, LabVIEW FPGA and MATLAB, where the digital frequency translation and baseband processing take place. Three independent I/Q signals are generated at the controller and passed to the dedicated reconfigurable RF signal generators (PXIe-5793), which were 
verified as having linear operation over the measurement range investigated. The configuration emulates a HetNet of three cells on different carrier frequencies. The RF outputs of the signal generators are combined (ZAPD-2-272-S+) and transmitted through a wideband antenna (UHALP-9108 A).

The RF digitizing single chain radio receiver is comprised of the proposed tri-band antenna, a digital storage oscilloscope (WaveRunner 640-Zi) acting as an RF digitizer, a reconfigurable tri-channel digital down-converter (DDC) and the baseband processors. The composite RF signal is directly digitized by the oscilloscope and the raw ADC samples are passed to the controller via a direct Ethernet link. Then digital down-conversion and baseband demodulation are executed, respectively, to retrieve the information bits at the controller.

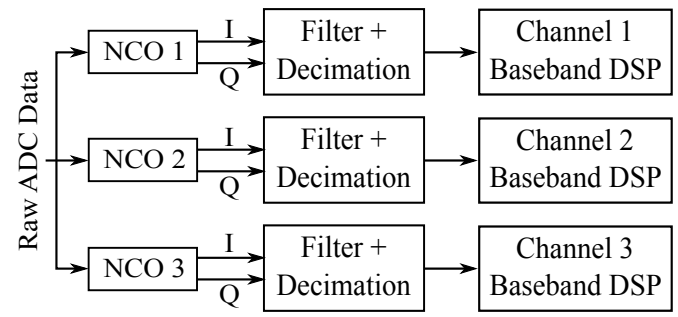

Fig. 10. Block diagram of a tri-channel reconfigurable DDC with baseband processing units.

The block diagram of a tri-channel DDC is shown in Fig. 10 together with the baseband processing units. DDC provides frequency conversion and decimation filtering of the desired bands before baseband demodulation takes place. The real digitized RF signal, in the form of ADC samples, is mixed and down-converted to baseband in-phase (I) and quadrature (Q) signals by three distinct digital synthesisers, known as numerically controlled oscillators (NCOs). The DDC was implemented as a direct or homodyne converter. Hence, the centre frequencies of the NCOs are set to equal the carrier frequencies of the three transmitted signals, correspondingly.
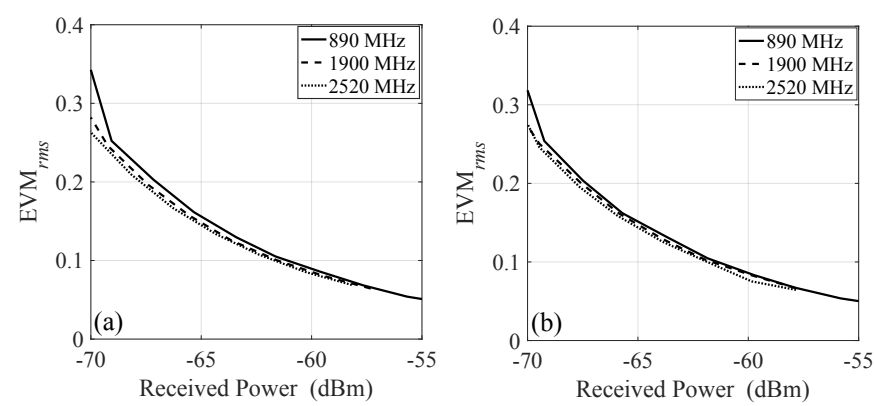

Fig. 11. Measured EVM $_{r m s}$ performance of a) concurrent multi-band and b) individual QPSK modulated single-carrier transmissions over the tri-band test-bed.

The baseband I/Q signal is passed through a cascaded integrated comb (CIC) decimation filter, which provides image and out-of-band rejection, as well as sample rate reduction to a predefined level. In this research, the DDC was implemented using a MATLAB digital signal processing (DSP) function, whereby the NCO centre frequencies, the CIC stopband frequencies and attenuations, and the decimation factors are pre- configured according to the bandwidths and carrier frequencies of the transmitted signals. The filtered, decimated baseband I/Q signals are then processed in LabVIEW whereby, for each frequency band, the timing, carrier and phase offsets are removed through the use of a synchronisation sequence and by locking to the carrier signal. Finally, pulse-shape matched filtering is performed before the received QPSK constellation $r m s$ error vector magnitude (EVM) is calculated using (2) [26]. In (2), $N$ is the number of samples received, $I$ and $Q$ are the nominal in-phase and quadrature values for QPSK, and $\tilde{I}$ and $\tilde{Q}$ are the received in-phase and quadrature values.

$$
\mathrm{EVM}_{r m s}=\sqrt{\frac{\frac{1}{N} \sum_{i=1}^{N}\left(I_{i}-\tilde{I}_{i}\right)^{2}+\left(Q_{i}-\tilde{Q}_{i}\right)^{2}}{\frac{1}{N} \sum_{i=1}^{N}\left(I_{i}^{2}+Q_{i}^{2}\right)}}
$$

\section{B. EVM Performance of Concurrent Transmissions}

In order to investigate the IBI, three independent QPSK modulated single-carrier signals centered at $890 \mathrm{MHz}, 1900$ $\mathrm{MHz}$ and $2520 \mathrm{MHz}$ were transmitted (at the same power), received and analyzed for their rms EVM. A signal bandwidth of $10 \mathrm{MHz}$ was used for each transmission, yielding a total system bandwidth of $30 \mathrm{MHz}$. Fig. 11(a) plots EVM versus received signal power for the three processed signals in concurrent transmission mode. The results in Fig. 11(a) show that the EVM performance, and hence the data transmission qualities, of the three bands are very similar. For QPSK, an EVM performance up to $20 \%$ is considered acceptable, which was achieved for a received power of $-68 \mathrm{dBm}$ in the test-bed with no data transmission errors.

To establish whether or not the concurrently received signals exhibit mutual interference, the EVM performances for the same QPSK single-carrier signals were measured separately and the results are plotted in Fig. 11(b). The curves for separately measured channels are almost identical to those for the concurrently measured channels, shown in Fig. 11(a). That is, the received signals in concurrent transmission mode do not experience appreciable mutual coupling when processed by the single chain receiver. Also, the rate of increase in EVM with decreasing received power is the same in concurrent and separate transmission modes.

\section{Resilience to Adjacent Blockers}

In this subsection, we consider unwanted or blocker signals, which may appear at adjacent frequencies to the wanted signals and, depending on their nature, could affect the signal quality of the wanted signals. Most blocker signals will be attenuated at the front-end by the filtering characteristics of the tunable antenna (see Fig.6). Nonetheless, the different bandwidths of each band at different tuning frequencies (see Table II) means that adjacent blockers could degrade system performance unless adequate filtering is included.

The adjacent blocker signals must be either filtered in the analogue domain (e.g. through a blocker resilient LNA) or must be filtered/attenuated through the digital CIC filters after the DDC. The LNA in the oscilloscope (WaveRunner 640-Zi) is wide-band, as required, and does not have blocker rejection 

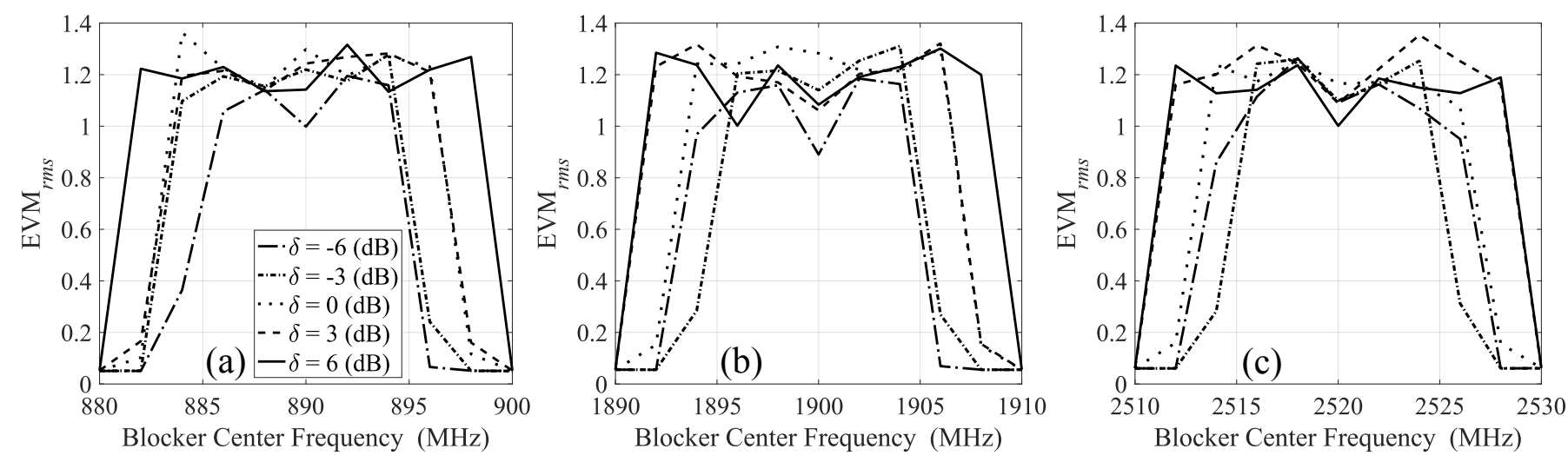

Fig. 12. The effect of adjacent/co-located SCM blockers on the EVM performance of the concurrent single-carrier transmissions at a) $890 \mathrm{MHz}$, b) 1900 $\mathrm{MHz}$ and c) $2520 \mathrm{MHz}$, measured on the test-bed.

capabilities. Therefore, in our test-bed adjacent signal rejection is provided by digital CIC filtering as well as the antenna.

To further characterize the CM-FARAD receiver, the EVM performance of each band was evaluated in the presence of single-carrier modulated (SCM) blocker signal located adjacent to or co-located with the wanted bands. The power of the blocker signals was varied such that the ratio of the received power of the blocker relative to the received power of the wanted signals changed from -6 to $+6 \mathrm{~dB}$. This ratio or the relative power of the blocker signal at the receiver is defined in (3), where $P_{\text {Blocker }}$ and $P_{\text {Signal }}$ are the blocker and signal powers at the receiver, respectively. The blocker signal was generated by another signal generator (SMBV100A) and transmitted by another wide-band antenna (UHALP-9108 A).

$$
\delta=10 \log _{10}\left(P_{\text {Blocker }} / P_{\text {Signal }}\right)
$$

Fig. 12 show the change in the EVM performance of the wanted signals as $\delta$ and the center frequency of the SCM blocker was changed, respectively. The SCM blocker was of the same type as the wanted signal, i.e. a $10 \mathrm{MHz}$ bandwidth single-carrier QPSK modulated signal. The results in Fig. 12 show that the EVM was not affected by the adjacent blocker for any of the considered $\delta$ values, provided the blocker signals were centered $10 \mathrm{MHz}$ or more away from the wanted signal. For this condition, the blocker and wanted signals do not overlap in frequency. Some spectral overlap can be tolerated for low power blocker signals. However, high power blockers affected the wanted signal when a spectral overlap of 1 to $2 \mathrm{MHz}$ was realised. Fig. 12 shows that the SCM blocker produced an EVM greater than unity when the blocker and wanted signals were co-located. For this high EVM, the wanted information bits were unrecoverable.

The results in Fig. 12 show that the combined antenna and digital CIC filtering effectively reject adjacent blocker interference for a $\delta$ up to $6 \mathrm{~dB}$. The results also show that a partially or fully co-located blocker increases the EVM of the wanted signals from 0.05 to 1.2. Investigation of adjacent channel interference with blocker signal powers leading to $\delta$ greater than $20 \mathrm{~dB}$ is a topic of further research in FARAD.

\section{COnClusions And Future Work}

The paper has presented a compact, separately tunable, triband antenna design for frequency agile, concurrent multiband, single chain radio transceivers. The antenna consists of three narrow slots fed by one microstrip line through electromagnetic coupling. The results show that the antenna can support three independently tunable operating bands simultaneously, over the frequency ranges $0.6-1.1 \mathrm{GHz}, 1.0$ $2.5 \mathrm{GHz}$ and $1.9-2.7 \mathrm{GHz}$. When digitally tunable capacitors were used in this design, from 0.51 up to $2.6 \mathrm{dBi}$ antenna gain improvement was obtained over a varactor diode based design. The varactor diode antenna based system level performance was also evaluated in a frequency agile, concurrent tri-band, direct RF digitizing, single chain radio receiver test-bed. For the parameters considered, the system level investigations demonstrated that an UE, using the proposed antenna, would incur negligible EVM degradation when processing concurrent wideband channels, thereby supporting a total system bandwidth of $30 \mathrm{MHz}$ using a single-chain radio receiver. By using the proposed antenna, the receiver also demonstrated strong adjacent blocker resilience when tested against modulated single carrier interferers. Future research will address the design and testing of a compact, independently tunable, pentaband antenna covering the frequency range from 0.6 to $6 \mathrm{GHz}$.

\section{ACKNOWLEDGEMENT}

This work was carried out in the FARAD project (https://www.commnet.ac.uk/groups/frequency-agile-radiofarad-project/), funded by the UK government under the EPSRC grant EP/M013723/1. The work is also supported by the UK Mobile Virtual Centre of Excellence (mVCE).

\section{REFERENCES}

[1] NGMN Alliance, "5G white paper," Next Generation Mobile Networks, White paper, 2015.

[2] J. He, P. Loskot, T. O'Farrell, V. Friderikos, S. Armour, and J. Thompson, "Energy efficient architectures and techniques for green radio access networks," in Communications and Networking in China (CHINACOM), 2010 5th International ICST Conference on. IEEE, 2010, pp. 1-6.

[3] H. Hamdoun, P. Loskot, T. OFarrell, and J. He, "Survey and applications of standardized energy metrics to mobile networks," Annals of telecommunications-annales des télécommunications, vol. 67, no. 3-4, pp. 113-123, 2012. 
[4] R. Singh, Q. Bai, T. O'Farrell, K. L. Ford, and R. J. Langley, "Demonstration of RF Digitising Concurrent Dual-Band Receiver for Carrier Aggregation over TV White Spaces," in 84th Vehicular Technology Conference (VTC2016-Fall), 2016 IEEE, Accepted for Publication, Sep 2016.

[5] D. Peroulis, K. Sarabandi, and L. P. B. Katehi, "Design of reconfigurable slot antennas," IEEE Transactions on Antennas and Propagation, vol. 53, no. 2, pp. 645-654, Feb 2005.

[6] K. Sakamoto, E. Nishiyama, and M. Aikawa, "Active microstrip planar antenna for frequency switching," Electronics and Communications in Japan (Part I: Communications), vol. 88, no. 11, pp. 1-8, 2005.

[7] L. Liu, J. Rigelsford, and R. Langley, "Tunable Multiband Handset Antenna Operating at VHF and UHF Bands," IEEE Transactions on Antennas and Propagation, vol. 61, no. 7, pp. 3790-3796, July 2013.

[8] N. Behdad and K. Sarabandi, "Dual-band reconfigurable antenna with a very wide tunability range," IEEE Transactions on Antennas and Propagation, vol. 54, no. 2, pp. 409-416, Feb 2006.

[9] K. K. Tsang and R. J. Langley, "Design of circular patch antennas on ferrite substrates," IEE Proceedings - Microwaves, Antennas and Propagation, vol. 145, no. 1, pp. 49-55, Feb 1998.

[10] L. Liu and R. J. Langley, "Liquid crystal tunable microstrip patch antenna," Electronics Letters, vol. 44, no. 20, pp. 1179-1180, September 2008.

[11] Z. H. Hu, C. T. P. Song, J. Kelly, P. S. Hall, and P. Gardner, "Wide tunable dual-band reconfigurable antenna," Electronics Letters, vol. 45, no. 22, pp. 1109-1110, October 2009.

[12] A. F. Sheta and M. A. Alkanhal, "Compact dual-band tunable microstrip antenna for gsm/dcs-1800 applications," IET Microwaves, Antennas Propagation, vol. 2, no. 3, pp. 274-280, April 2008.

[13] B. Avser and G. M. Rebeiz, "Tunable Dual-Band Antennas for 0.71.1-GHz and 1.7-2.3-GHz Carrier Aggregation Systems," Antennas and Propagation, IEEE Transactions on, vol. 63, no. 4, pp. 1498-1504, 2015.

[14] A. Boukarkar, X. Q. Lin, and Y. Jiang, "A dual-band frequency-tunable magnetic dipole antenna for wimax/wlan applications," IEEE Antennas and Wireless Propagation Letters, vol. 15, pp. 492-495, 2016.

[15] X. Chen, W. Chen, F. M. Ghannouchi, Z. Feng, and Y. Liu, "Enhanced Analysis and Design Method of Concurrent Dual-Band Power Amplifiers With Intermodulation Impedance Tuning," IEEE Transactions on Microwave Theory and Techniques, vol. 61, no. 12, pp. 4544-4558, Dec 2013.

[16] H. Hashemi and A. Hajimiri, "Concurrent dual-band cmos low noise amplifiers and receiver architectures," in VLSI Circuits, 2001. Digest of Technical Papers. 2001 Symposium on. IEEE, 2001, pp. 247-250.

[17] J. Borremans, G. Mandal, V. Giannini, B. Debaillie, M. Ingels, T. Sano, B. Verbruggen, and J. Craninckx, "A $40 \mathrm{~nm}$ CMOS 0.4-6 GHz receiver resilient to out-of-band blockers," IEEE Journal of Solid-State Circuits, vol. 46, no. 7, pp. 1659-1671, July 2011.

[18] J. Marttila, M. Allén, and M. Valkama, "Frequency-agile multiband quadrature sigma-delta modulator for cognitive radio: Analysis, design and digital post-processing," Selected Areas in Communications, IEEE Journal on, vol. 31, no. 11, pp. 2222-2236, 2013.

[19] National Instruments, "Embedded Signal Generator, PXIe-5793." [Online]. Available: http://www.ni.com/pdf/manuals/373949b.pdf

[20] — ,Embedded Signal Generator, PXIe-5791." [Online]. Available: http://www.ni.com/pdf/manuals/373845d.pdf

[21] Lime Microsystems, "Multi-band multi-standard Transceiver IC, LMS6002." [Online]. Available: http://www.limemicro.com/products/ field-programmable-rf-ics-1ms6002d/

[22] —, "Multi-band multi-standard Transceiver IC, LMS7002M." [Online]. Available: http://www.limemicro.com/products/ field-programmable-rf-ics-1ms $7002 \mathrm{~m} /$

[23] R. Garg, Microstrip Antenna Design Handbook, ser. Antennas and Propagation Library. Artech House, 2001.

[24] C. A. Balanis, Antenna Theory: Analysis and Design, 3rd Edition, 3rd ed. WILEY, 2012.

[25] Y. Li, Z. Zhang, Z. Feng, and M. F. Iskander, "Design of penta-band omnidirectional slot antenna with slender columnar structure," IEEE Transactions on Antennas and Propagation, vol. 62, no. 2, pp. 594601, Feb 2014.

[26] "IEEE Standard for Local and Metropolitan Area Networks Part 16: Air Interface for Fixed Broadband Wireless Access Systems," IEEE Std 802.16-2004 (Revision of IEEE Std 802.16-2001), pp. 01-857, 2004.

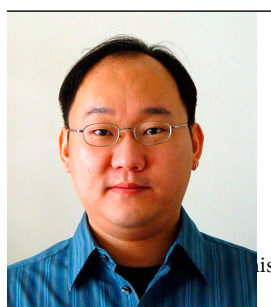

Qiang Bai received the M.S. degree in electronics engineering from the University of Kent, Canterbury, U.K., in 2006 and the Ph.D. degree in electronic engineering from the University of Sheffield, U.K., in 2012. He is currently working as research associate at the Department of Electronic and Electrical Engineering, University of Sheffield, Sheffield, U.K. His research interests include MIMO antennas, re-

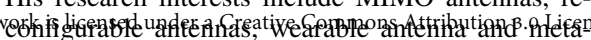

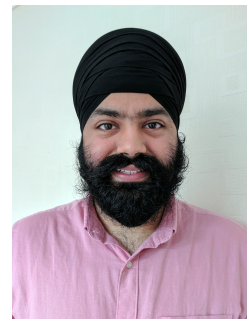

Ravinder Singh (M'15-S'13) graduated with a $\mathrm{PhD}$ and First Class Honours M.Eng degree in Electronic and Communications Engineering from the University of Sheffield, U.K. in 2016 and 2011, respectively. He is currently a post-doctoral research associate at the same university working on the frequency agile radio (FARAD) project. His research interests include PHY techniques for mobile and wireless communications, visible light communication, software-defined radio, cognitive radio and reconfigurable concurrent multi-band transceivers.

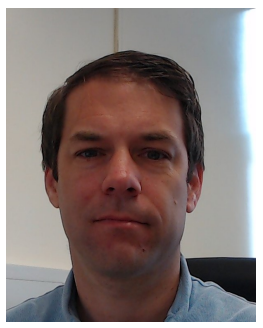

Kenneth Lee Ford (M07-SM10) received the B.Eng. and Ph.D. degrees in electronic engineering from the University of Sheffield, Sheffield, U.K., in 1998 and 2003, respectively. In 2001, he joined the Stealth Materials Department, Advanced Technology Centre, BAE SYSTEMS, London, U.K., before returning to the University of Sheffield in 2005 as a Lecturer of Communications. He was promoted to Senior Lecturer in 2012. His research interests include reconfigurable antennas, miniaturized antennas, metamaterials, propagation in the built environment, and electromagnetic structures for biomedical applications.

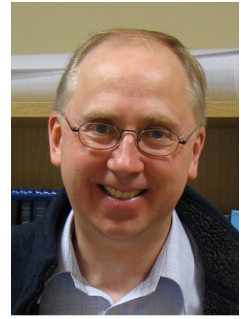

Timothy O'Farrell (M'91) is Chair Professor in Wireless Communications at the University of Sheffield, UK. He researches wireless communication systems specialising in physical layer signal processing, radio resource management and wireless network planning. He has pioneered research on energy efficient mobile cellular communications, the mathematical modeling of CSMA based MAC protocols for $\mathrm{WiFi}$, coded modulation techniques for optical wireless communication systems and spreading sequence design for CDMA wireless networks. $\mathrm{He}$ is an entrepreneur, being the cofounder and CTO of Supergold Communication (2000-2007), a start-up that participated in the standardisation of IEEE $802.11 \mathrm{~g}$ with the MBCK proposal. In the framework of Mobile VCE (mVCE), Professor O'Farrell was the Academic Coordinator of the Core 5 Green Radio project (2009-2012) and a leader in establishing energy efficiency as a global research field in wireless communication systems. He has managed 24 major research projects as principal investigator; published 312 journal and conference papers, book chapters, patents and technical reports; and has participated in standards, consultancy and expert witness activities within the wireless sector. Currently, Professor OFarrell is leading the FARAD project and the UK Research Strategy Community Organisation in Communications, Mobile Computing and Networking within the EPSRC portfolio (www.commnet.ac.uk). Professor O'Farrell is a Director of the mVCE, a Chartered Engineer and a member of the IET and IEEE.

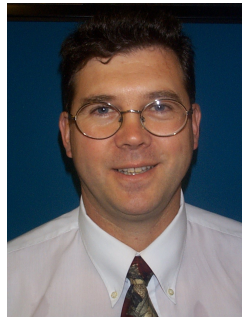

Richard J. Langley (M97-SM12) has BSc and PhD degrees from the University of Kent, UK. After spending some time working on communications satellites at Marconi Space Systems in the 1970s he became a lecturer at the University of Kent in 1979. Richard was promoted to a personal Chair in Antenna Systems in 1994 and was Honorary Editor of IEE Proceedings Microwaves, Antennas and Propagation from 1995-2003. In 1997 he founded the European Technology Centre for Harada Industries Japan, the worlds largest supplier of automotive antennas. The centre researches and develops advanced hidden antenna systems for the global automotive market including radio, telephone and navigation systems. After successfully building up the technology and business he rejoined academic life in 2003. Professor Langley is now Head of the Communications Research Group at the University of Sheffield.

His main research is in the fields of automotive antennas, propagation in the built environment, frequency selective surfaces, electromagnetic band gap materials and applications, multi-function antenna systems and reconfigurable antennas. He initiated the setting up of the Wireless Friendly Building Forum in 2009 to address the problems of wireless signal propagation in buildings and the built environment. Richard has published over 400 papers in international

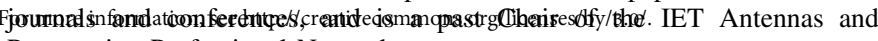
Propagation Professional Network 